\title{
Thin film free-standing PEDOT:PSS/SU8 bilayer microactuators
}

S Taccola, F Greco, B Mazzolai, V Mattoli and Edwin J ager

The self-archived postprint version of this journal article is available at Linköping University Institutional Repository (DiVA):

http:// urn.kb.se/ resolve?urn=urn:nbn:se:liu:diva-102387

N.B.: When citing this work, cite the original publication.

Taccola, S, Greco, F, Mazzolai, B, Mattoli, V, J ager, E., (2013), Thin film free standing

PEDOT:PSS/ SU8 bilayer microactuators, J ournal of Micromechanics and Microengineering, 23(11),

117004. https:// doi.org/ 10.1088/ 0960-1317/23/ 11/117004

Original publication available at:

https:// doi.org/ 10.1088/ 0960-1317/ 23/ 11/117004

Copyright: IOP Publishing (Hybrid Open Access)

http:// www.iop.org/ 


\title{
Thin film free standing PEDOT:PSS/SU8 bilayer microactuators
}

\author{
S Taccola ${ }^{1}$, F Greco ${ }^{1}$, B Mazzolai ${ }^{1}$, V Mattoli ${ }^{1, *}$ and E W H Jager ${ }^{2, *}$ \\ ${ }^{1}$ Center for Micro-BioRobotics @SSSA, Istituto Italiano di Tecnologia (IIT), Viale \\ Rinaldo Piaggio 34 Pontedera, 56025, Italy \\ ${ }^{2}$ Biosensors and Bioelectronics Centre, Department of Physics, Chemistry and \\ Biology, Linköping University, 58183 Linköping, Sweden \\ *Shared senior authorship \\ E-mail: silvia.taccola@iit.it
}

\begin{abstract}
Several smart active materials have been proposed and tested for the development of microactuators. Among these, conjugated polymers are of great interest because miniaturization improves their electrochemical properties such as increasing speed and stress output of microactuators, with respect to large scale actuators. Recently we developed a novel fabrication process to obtain robust large area, free standing, conductive ultra-thin films made of the conjugated polymer poly ( 3 , 4-ethylenedioxythiophene) doped with the polyanion poly(styrenesulfonate) (PEDOT:PSS). These conductive free-standing nanofilms, with thickness ranging between few tens to several hundreds of $\mathrm{nm}$, allow the realisation of new all-polymer microactuators using facile microfabrication methods. Here, we report a novel processing method for manufacturing all polymer electrochemical microactuators. We fabricated and patterned free-standing PEDOT:PSS/SU8 bilayer microactuators in the form of microfingers of a variety of lengths using adapted microfabrication procedures. By imposing electrochemical oxidation/reduction cycles on the PEDOT:PSS we were able to demonstrate reversible actuation of the microactuators resulting in bending of the microfingers. A number of possible applications can be envisaged for these small, soft actuators such as microrobotics and cell manipulation.
\end{abstract}

\section{Introduction}

Conjugated polymers (CP) like polypyrrole (PPy), polyaniline (PANI), and poly(3,4-ethylenedioxythiophene) (PEDOT), which undergo volume changes during electrochemical oxidation and reduction, are excellent materials for actuators [1-4]. The volume change is caused by the diffusion of ions and solvent into the film upon electrochemical oxidation or reduction. For PEDOT:PSS (PEDOT doped with the polyanion poly(styrenesulfonate)) this process can be described according to equation (1).

$$
\text { PEDOT }^{+}: \text {PSS }^{-}+\mathrm{e}^{-}+\mathrm{M}^{+} \text {(aq) <-> } \text { PEDOT }^{0}: \text { PSS }^{-}{ }^{+} \quad \text { (eq. 1) }
$$

That is upon electrochemical reduction of PEDOT:PSS from the oxidised state, cations $\mathrm{M}^{+}$such as $\mathrm{Na}^{+}$enter the matrix and the film swells. Upon oxidation the opposite occurs. This redox process is completely reversible. Since the volume change and thus actuation is based on diffusion, the use of thick films can result in slow responses thus limiting the performance and restricting the applicability of CP actuators. As the size is reduced by using thinner films, the effect of ion diffusion and resistance $\times$ capacitance (RC) time constant are reduced so that an increased speed of response compared to large-scale actuators is expected [5]. CP microactuators can find many useful applications, particularly in cell biology and biomedicine. They have been proposed for the manipulation of biological objects, such as single cells, or applied in microfluidic pumps and valves for drugs delivery or bioanalysis [6]. In microsystems, such microactuators can be used as hinges from which more complex systems can be built, such as self-assembling boxes [7], cell-clinics [8], 
and microrobots [9]. CP actuators in the form of free-standing films have mainly been based upon PPy, while less research has been undertaken using PEDOT. One of the reasons for this can be found in the lower strain (1-4\%) generated by PEDOT films, compared with PPy films (over 10\% strain). On the other hand, better electrochemical stability for PEDOT films compared with PPy films has been found [10]. Moreover, the PPy and PEDOT used in the fabrication of microactuators are commonly produced in situ, starting from a monomer solution using either electrochemical $[3,4,11]$ or chemical synthesis [12] which is time consuming and may result in less reproducible films. On the contrary, deposition of the electroactive conducting polymer layer by spin-coating results in faster deposition and well controlled, reproducible thickness. In this framework, PEDOT:PSS is particularly attractive because it is commercially available in the form of a ready-to-use waterborne dispersion.

Here we present a novel all polymer microactuator based on free-standing conductive nanofilms of PEDOT:PSS deposited by spin-coating. Recently we developed a novel, fast, and simple fabrication process for obtaining free-standing conductive nanofilms made of PEDOT:PSS [13]. These nanofilms had a thickness ranging between few tens to several hundreds of $\mathrm{nm}$. This opens up the possibility of using such freestanding PEDOT:PSS nanofilms to realize new all polymer electrochemical microactuators using facile microfabrication methods.

\section{Experimental}

\subsection{Fabrication of PEDOT:PSS/SU8 bilayer microactuators}

PEDOT:PSS nanofilms were obtained by spin-coating of a commercially available PEDOT:PSS aqueous dispersion (Clevios ${ }^{\mathrm{TM}}$ PH1000, 1:2.5 PEDOT : PSS ratio, Heraeus GmbH) following the procedure described in detail in [13]. Briefly, a thin film of poly(dimethylsiloxane) (PDMS, Sylgard 184 silicone elastomer base and curing agent, Dow Corning Corporation) was deposited by spin-coating onto silicon substrates (400 $\mu \mathrm{m}$ thick, $\mathrm{p}$ type, boron doped, $<100>$, Si-Mat Silicon Materials). In order to obtain a thin elastomer film (thickness $t \approx 800 \mathrm{~nm}$ ), PDMS (10:1 ratio of base elastomer to curing agent) was diluted with $n$-hexane (electronic use grade, $97 \%$, Acros Organics) by $15 \%$ in weight. The diluted PDMS solution was spin-coated onto Si substrates for $150 \mathrm{~s}$ at a speed of $6000 \mathrm{rpm}$ and then cured at $T=95^{\circ} \mathrm{C}$ for $60 \mathrm{~min}$ in an oven. A subsequent air plasma treatment (Harrick PDC-002 Plasma Cleaner, HarrickPlasma) was applied for $30 \mathrm{~s}$ at $5 \mathrm{~W}$ in order to temporally improve the wettability of the pristine PDMS surface, to allow the spreading of the PEDOT:PSS water dispersion and thus the formation of a homogeneous film by spincoating.

In order to increase the polymer concentration, the PEDOT:PSS aqueous dispersion (solid content 1.0 to $1.3 \%$ ) was filtered (Minisart ${ }^{\circledR}$, average pore size $1.20 \mu \mathrm{m}$, Sartorius) and reduced to $50 \%$ by weight by heating it at $\mathrm{T}=80^{\circ} \mathrm{C}$ in order to evaporate the water. The concentrated PEDOT:PSS dispersion was then spin-coated over the cured PDMS film at $2000 \mathrm{rpm}$ for $60 \mathrm{~s}$. Then, the samples underwent a thermal treatment $\left(1 \mathrm{~h} ; T=170^{\circ} \mathrm{C}\right)$, making the conductive polymer nanofilms resistant to water.

The feasibility of realizing a new electrochemical microactuator based on PEDOT:PSS nanofilms was investigated using the commonly used configuration of the bending bilayers. In order to realize this kind of devices, the PEDOT:PSS nanofilm (the electrochemically active layer) must be coupled to a passive layer and then patterned in order to produce rectangular beams with desired lengths (L) and widths (W). In general, there is considerable interest in patterning techniques that allow well-defined geometric features to be produced in organic thin film structures at near micrometer resolution, reproducibly and at low cost. Photolithography was used to pattern because, if carried out in a non-destructive manner, it would be the preferred method for patterning functional organic materials, due to its high resolution, high-throughput parallel nature, its scalability to large-area substrates, and its use of established equipment and expertise.

SU-8 is a high contrast, epoxy-based negative photoresist widely employed in the fabrication of microelectrical-mechanical systems (MEMS). In addition to its use as negative photoresist, SU-8 also possesses a number of properties that make it attractive as a structural material in microfabricated devices. SU-8 is biocompatible, rigid, as well as thermally and chemically stable. Therefore, SU-8 has been selected to pattern by photolithography the PEDOT:PSS nanofilm as well as to function as the structural passive layer in a bilayer PEDOT:PSS/SU8 microactuator. A proper formulation of SU-8 with thinner was selected in order to obtain a sub-micrometer thick layer of SU-8. 
Before spin-coating SU-8 on the surface of the PEDOT:PSS nanofilm, the sample was immersed in $1 \mathrm{M} \mathrm{NaCl}$ solution in order to promote the ion exchange between protons (mobile counter ions of the anionic immobile sulphonic acid groups of PSS) and monovalent cations $\left(\mathrm{Na}^{+}\right)$. The ion exchange is necessary because of the acidity of PEDOT:PSS; without this precaution, crosslinking of SU-8 would be initiated by protons on the surface before UV exposure. The samples were then rinsed with deionized water, dried by nitrogen blowing, baked on a hotplate at $100 \mathrm{C}$ for $10 \mathrm{~min}$ (dehydration), and finally exposed to air plasma for $60 \mathrm{~s}$ at $5 \mathrm{~W}$. Free-standing PEDOT:PSS/SU8 bilayer microactuators in the form of microfingers were fabricated by depositing a patterned thin layer of SU-8 (SU-8 2002/cyclopentanone 1:2 in vol., Microchem, spin-coated at $500 \mathrm{rpm}$ for $10 \mathrm{~s}$, then $5000 \mathrm{rpm}$ for $30 \mathrm{~s}$ ) on PEDOT:PSS nanofilms. Thereafter the PEDOT:PSS was wet chemically etched in a NaClO solution in water (2\%(vol:vol), Sigma-Aldrich) for $10 \mathrm{~s}$ (overoxidation), followed by a rinse in DI water, isopropyl alcohol (IPA) and once again DI water. Thereafter a support layer was deposited by drop casting a $10 \mathrm{wt}$. \% poly(vinylalcohol) aqueous solution (PVA, average molecular weight $\mathrm{M}_{\mathrm{W}}=30 \mathrm{kDa}$, Sigma-Aldrich) over the samples and dried overnight. The microactuators were then peeled off from the substrate. Figure 1 schematically summarizes the steps of the developed fabrication procedure.

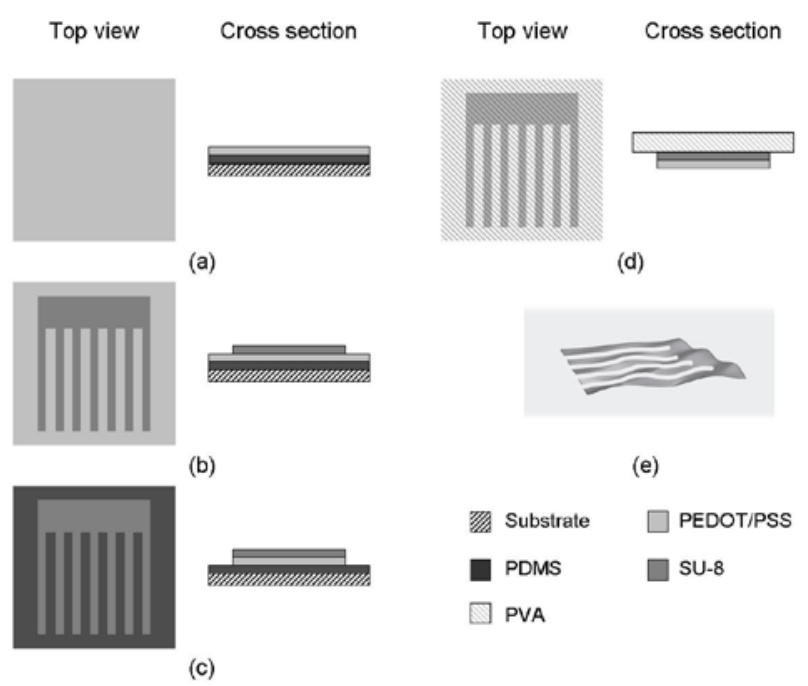

Figure 1. (a) Deposition of PEDOT:PSS on the PDMS substrate layer; (b) deposition and photolithographic patterning of the SU-8 layer; (c) wet chemical etching of PEDOT:PSS giving the final microactuator structure; (d) peeling of the bilayer microactuator using a PVA support layer; (e) free-standing PEDOT:PSS/SU8 microactuator floating in water after dissolving PVA.

In order to provide the electrical signals to the material, electrodes and wiring were added on the microactuators in the dry state, while they were still supported by the PVA layer. A gold layer $(\approx 500 \mathrm{~nm}$ thick) was deposited at the base of the microfingers by DC sputtering (Q150T S Sputter Coater, Quorum Technologies Ltd.) onto the PEDOT:PSS surface through a shadow mask (electrode size $5 \mathrm{~mm} \times 5 \mathrm{~mm}$ ). An insulated copper wire was then attached to the gold electrode with silver paint and protected with acrylic glue.

The PEDOT:PSS/SU8 microactuators were finally released by dissolving the PVA layer in DI water.

The thickness of the PEDOT:PSS nanofilms and of the SU-8 layer were measured using a KLA Tencor stylus Profiler. The sheet resistance of the PEDOT:PSS nanofilm was evaluated by using a home-made four probes apparatus, described in detail in [13].

\subsection{Electrochemical and electromechanical responses}

The electrochemical response of the actuators was investigated using a Gamry Reference 600 Potentiostat/Galvanostat/ZRA operated with Gamry PHE200 software. PEDOT:PSS/SU8 bilayer microactuators were connected to the working electrode (WE) in a three electrode cell containing a reference electrode $\mathrm{Ag} / \mathrm{AgCl}(\mathrm{RE})$ and a platinum wire as the counter electrode (CE). Cyclic voltammetry (CV) experiments were then carried out in the voltage range from +0.4 to $-0.8 \mathrm{~V}$ at a scan rate of $20 \mathrm{mVs}^{-1}$ in a 
0.1 M NaDBS aqueous solution (Sigma-Aldrich). The electromechanical response of the actuators was investigated by observing and video recording microactuators movements during CV experiments with a digital optical microscope (Hirox KH7700 digital microscope, Hirox Co Ltd.).

\section{Results and discussions}

\subsection{Bilayer microactuators fabrication}

As a proof of concept demonstrating the feasibility of the fabrication process and the actuation principle, microactuators having the following geometric parameters were built: PEDOT:PSS nanofilms with thickness $220 \mathrm{~nm}$; SU-8 layer thickness $340 \mathrm{~nm}$; width of the beams $150 \mu \mathrm{m}$.

Figure 2(a) and 2(b) show the resulting microactuators: each unit is composed of 6 microfingers with decreasing length (from the left to the right the ratio $W: L$ has the following values: 1:15, 1:12.5, 1:10, 1:7.5, 1:5, 1:2.5). It showed that attaching a wire with the silver paint directly on the PEDOT:PSS surface easily damaged the surface itself, resulting in a weak electrical contact. Therefore, gold was sputtered on top of the PEDOT:PSS at the base of the actuators to establish a good electrical contact between the PEDOT:PSS nanofilm and connecting the wire. Figure 2(c) and (d) show the gold contact on the PEDOT:PSS side of the bilayer microactuator and the electrical contact of a PEDOT:PSS/SU8 bilayer microactuator with the wire. From four probe measurements, the sheet resistance of PEDOT:PSS nanofilms was determined to be $23 \mathrm{k} \Omega$ square $^{-1}$.
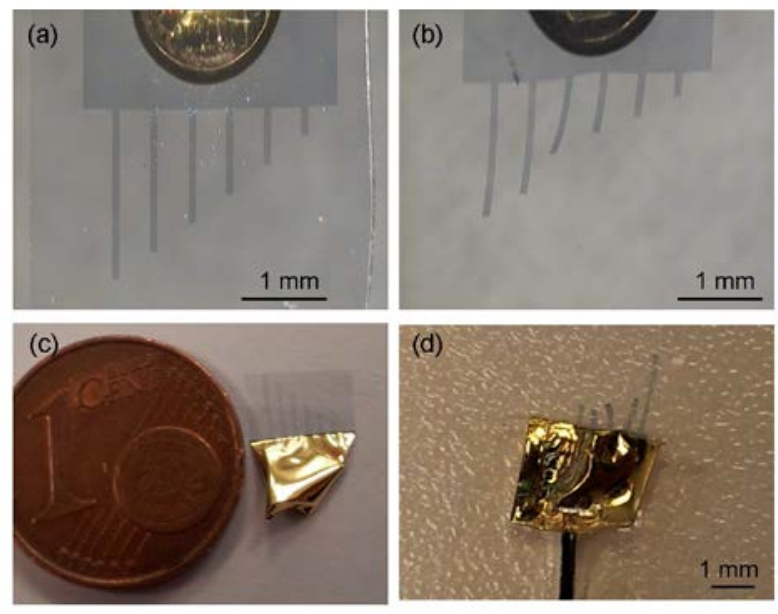

Figure 2. Patterned PEDOT:PSS/SU8 microactuator (a) in the dry state, supported by the PVA layer; (b) floating in the water after dissolving PVA; (c) the sputtered gold contact and (d) the electrical contact (in water).

After dissolving the PVA layer in DI water, the individual fingers of the actuator were released (Figure $2 \mathrm{~b}$ and 2d). As can be seen, there existed some initial bending of the fingers. This initial bending and curling of the microactuator in the pristine state could be a result of both residual mean stress and residual stress gradient in the microfingers. In general, non-uniform processing conditions throughout the beam thickness, material deposition temperatures, and the mismatch of the coefficients of thermal expansion (CTE) between material layers are key contributing factors to residual and interface stresses [14]. In particular, these effects become particularly important as film thickness is reduced [15]. In order to minimize residual stress, a detailed investigation of the effect of processing conditions (e.g. exposure doses, post-exposure baking time and temperature, etc) on the initial bending of the microfingers is currently underway and will be reported in future work.

\subsection{Electrochemical properties}

As reported in the Experimental Section, the electrochemical properties of the PEDOT:PSS/SU8 bilayer microactuators were examined with cyclic voltammetry (CV). We found that the shape of the voltammetric curve did not show any substantial changes compared to no-treated PEDOT:PSS nanofilms, with redox 
peaks located at their normal positions (i.e. the reduction peak at $-0.25 \mathrm{~V}$ and the oxidation peak at about $0 \mathrm{~V}$ vs Ag/AgCl, figure 3), confirming that the electrochemical behaviour of PEDOT:PSS was not influenced by processing.

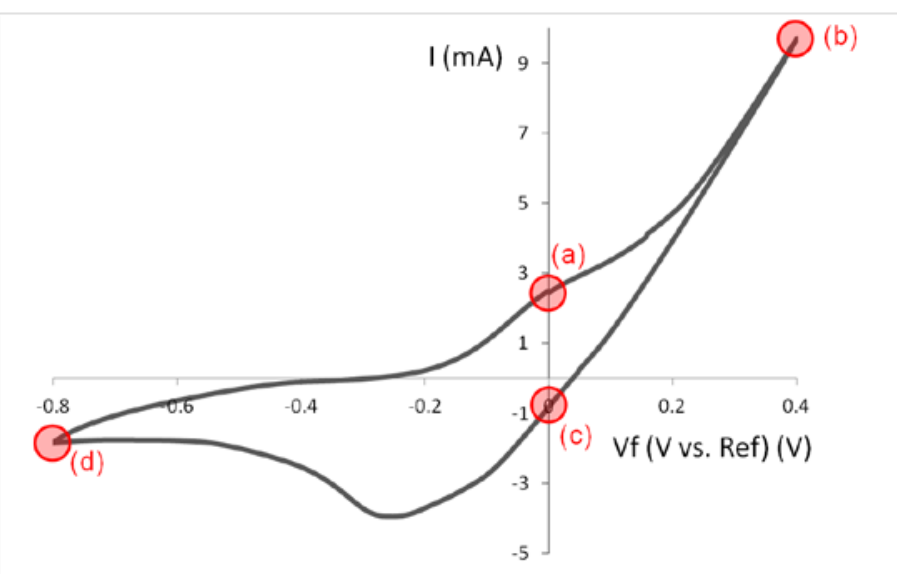

Figure 3. Cyclic voltammetry of PEDOT:PSS/SU8 bilayer microactuator in $0.1 \mathrm{M}$ NaDBS electrolyte. Scan rate $20 \mathrm{mVs}^{-1}$. Letters indicate to four different states of the actuator: (a) pristine state (V=0 V); (b) oxidised state $(\mathrm{V}=+0.4 \mathrm{~V})$; (c) back to $\mathrm{V}=0 \mathrm{~V}$; $(\mathrm{d})$ reduced state $(\mathrm{V}=-0.8 \mathrm{~V})$ and correspond to figure $4 \mathrm{a}-\mathrm{d}$.

\subsection{Electrochemical actuation}

Figure 4 shows an example of the microactuators movement observed during CV, with PEDOT:PSS lying on the top side of the bilayer. A displacement, both bending and curling, of the microfingers from the starting position ( $\mathrm{V}=0 \mathrm{~V}$, figure $4(\mathrm{a})$ ), is observed during oxidation of the PEDOT:PSS ( $\mathrm{V}=0.4 \mathrm{~V}$, figure $4(\mathrm{~b})$ ). A displacement in the opposite direction is observed during reduction, recovering the initial position at $\mathrm{V}=0 \mathrm{~V}$ (figure 4(c)) and further bending in the fully reduced state ( $\mathrm{V}=-0.8 \mathrm{~V}$, figure $4(\mathrm{~d})$ ). The bending and curling motion follows the pattern that would be expected from the electrochemical reaction (1): contracted/bent in the oxidized state and expanded/straightened in the reduced state. 

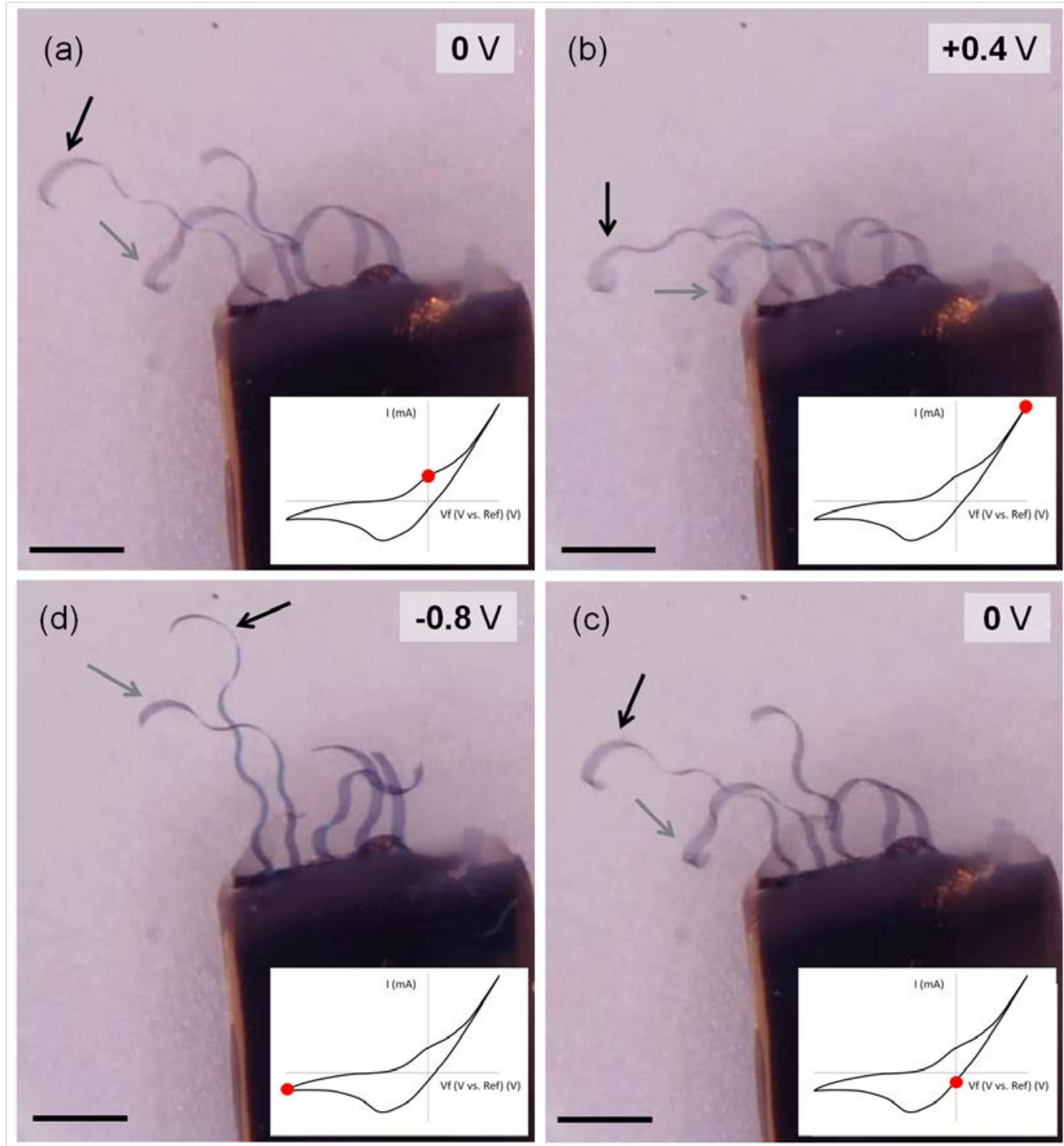

Figure 4. Top view of the movement of PEDOT:PSS/SU8 microactuators during CV (showed as a reference in the inset): (a) starting position of microfingers $(\mathrm{V}=0 \mathrm{~V})$; (b) bending caused by oxidation $(\mathrm{V}=+0.4 \mathrm{~V})$; (c) recovery of initial position at $\mathrm{V}=0 \mathrm{~V}$; (d) opposite displacement caused by reduction $(\mathrm{V}=-0.8 \mathrm{~V})$. Scale bar is $1 \mathrm{~mm}$.

As evidenced in figure 5, fingers displacements were on the order of hundreds to thousands of microns (table 1). As can be seen the general trend is that the longer fingers show larger tip displacement, but not all fingers follow this consideration. This is possibly caused by the fact that some parts of the fingers twist instead of curl, thus reducing the tip displacement. Moreover, together with displacement, a curling movement of all the fingers was observed, with a decrease of curvature radius $r$ of the tip during oxidation and an increase during reduction, again following eq. 1 . An estimate of the change in the radius of curvature is given in figure 5 for the tip of finger number 1 as an example. In this case, the curvature $k=1 / r$ changed from about $6 \mathrm{~mm}^{-1}(\mathrm{~V}=0 \mathrm{~V})$ to about $10 \mathrm{~mm}^{-1}$ in the fully oxidised state $(\mathrm{V}=+0.4 \mathrm{~V})$ and to about $4 \mathrm{~mm}^{-1}$ in the fully reduced state $(\mathrm{V}=-0.8 \mathrm{~V})$. These results confirm the expected behaviour based on the known actuation mechanism of PEDOT:PSS. Indeed, in the oxidized state a contraction of PEDOT:PSS occurs due to the ejection of $\mathrm{Na}^{+}$ions thus resulting in a bent configuration of microfingers. On the reverse, reduction causes expansion due to the insertion of $\mathrm{Na}^{+}$ions, with corresponding bending in the opposite direction. 

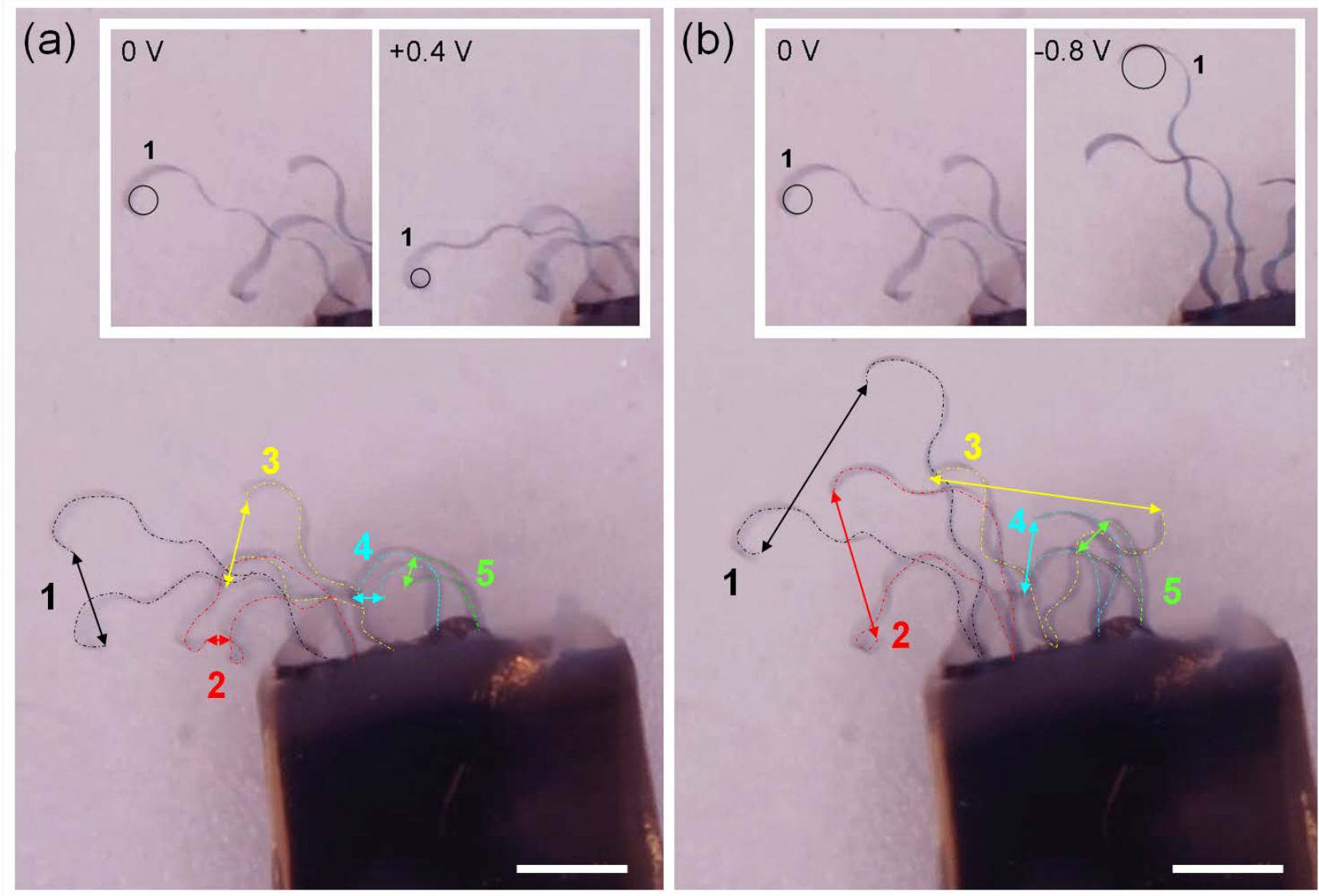

Figure 5. Top view overlay images of the starting positions of the microfingers and their positions (a) in the oxidized and (b) in the reduced state. For each finger (evidenced with the help of different colour dotted lines), the displacement has been measured as the distance between tip of finger. In the inset is reported the measurement of the radius of curvature for the tip of finger number 1 . Scale bar $1 \mathrm{~mm}$.

Table 1. Fingers displacement during CV.

\begin{tabular}{cccc}
\hline Finger & Length $(\mu \mathrm{m})$ & $\begin{array}{c}\text { Displacement caused } \\
\text { by oxidation }(\mu \mathrm{m})\end{array}$ & $\begin{array}{c}\text { Displacement caused } \\
\text { by reduction }(\mu \mathrm{m})\end{array}$ \\
\hline 1 & 2250 & 760 & 1760 \\
2 & 1875 & 220 & 1380 \\
3 & 1500 & 780 & 2090 \\
4 & 1125 & 280 & 660 \\
5 & 750 & 300 & 400 \\
\hline
\end{tabular}

With regards to the actuation time, the results showed in figure 4 were obtained by operating the microactuators using CV. In this case, the total time of actuation from $0.4 \mathrm{~V}$ to $-0.8 \mathrm{~V}$ was $60 \mathrm{~s}$, as imposed by the set scan rate of $\mathrm{CV}(20 \mathrm{mV} / \mathrm{s})$. On the other hand, operating microactuators in chronoamperometry (setting a voltage step from $+0.4 \mathrm{~V}$ to $-0.8 \mathrm{~V}$ and back to $+0.4 \mathrm{~V}$ ) a full displacement between the two end positions was achieved in $1-2 \mathrm{~s} \mathrm{~d}$.

The large displacement, including bending along the full length of the fingers, showed that the conductivity of the electroactive PEDOT:PSS layer was sufficiently high to allow for the redox reaction to occur in all of the PEDOT:PSS. No reduction of actuation was observed due to an iR drop in the PEDOT film [16]. Thus a conducting layer, like gold, deposited between the passive layer and conducting polymer, which is common practice in CP microactuators, is indeed not needed, which was one the goals of this work: microfabricating an all polymer microactuator.

The obtained results are encouraging, despite the fact that the geometrical parameters of microactuators were not fully optimized. Indeed, in general, the geometrical parameters that impact the performance of a beam-shaped bilayer microactuator are: the length and width of the beam (W:L ratio), the total thickness of the bilayer, the ratio of the active to passive layer thicknesses, and the ratio of the Young's moduli of the two 
materials [17]. Using the proposed fabrication method, the geometrical parameters of the PEDOT:PSS/SU8 microactuators could be easily controlled, looking for the configuration for achieving optimal actuator performance: microactuators with different size and shape could be obtained using dedicated photolithography masks; the thickness of the SU-8 layer could be varied by changing the level of dilution of SU-8 and the spin-coating speed during the photoresist deposition; the thickness of the PEDOT:PSS nanofilms could also be varied by changing the fabrication parameters (e.g. spin-coating speed, polymer concentration) in order to obtain nanofilms with controlled thicknesses.

\section{Conclusions}

We have developed a novel, all polymer PEDOT:PSS based microactuator. We demonstrated, for the first time, the use of the spin-coating technique for depositing the electroactive $\mathrm{CP}$ layer, thus allowing for reproducible batch fabrication.

In this study, the possibility to use the electrochemical properties of PEDOT:PSS nanofilms to realize ultra-thin microactuators has been demonstrated and characterized. Microactuators with different size and shape were obtained using dedicated photolithography masks. Here we presented preliminary results demonstrating simple bending actuation of a microfinger structure. We are currently continuing this work extending the actuation performance and evaluating the actuator performances (e.g. radius of curvature), as well as correlate the data to existing models [17-19], which will be reported in future work.

\section{Acknowledgment}

EWHJ wishes to express his gratitude to Prof Anthony Turner, LiU. Funding has been supplied by European Science Foundation COST Action MP1003 ESNAM (European Scientific Network for Artificial Muscles), Linköping University and IIT.

\section{References}

[1] Pei Q and Inganäs O 1992 Adv. Mater. 4 277-278

[2] Otero T F and Sansiñena J M 1998 Adv. Mater. 10 491-494

[3] Smela E, Inganäs O and Lundström I 1993 J. Micromech. Microeng. 3 203-205

[4] Jager E W H, Smela E and Inganäs O 2000 Science 290 1540-45

[5] Baughman R H 1996 Synth. Met. 78 340-353

[6] Smela E 2003 Adv. Mater. 15 481-494

[7] Smela E, Inganäs O and Lundström I 1995 Science 268 1735-38

[8] Jager E W H, Immerstrand C, Petersson K H, Magnusson K E, Lundström I and Inganäs O 2002 Biomedical Microdevices 4 177-187

[9] Jager E W H, Inganäs O and Lundström I 2000 Science 288 2335-38

[10] Kiefer R, Bowmaker G A, Kilmartin P A and Travas-Sejdic J 2010 Electrochim. Acta 55 681-688

[11] Alici G, Devaud V, Renaud P and Spinks G 2009 J. Micromech. Microeng. 19 025017/1-025017/9

[12] Khaldi A, Plesse C, Soyer C, Cattan E, Vidal F, Legrand C and Teyssié D 2011 Appl. Phys. Lett. 98 164101-04

[13] Greco F, Zucca A, Taccola S, Menciassi A, Fujie T, Haniuda H, Takeoka S, Dario P and Mattoli V 2011 Soft Matter 7 10642-51

[14] Sameoto D, Tsang S H, Foulds I G, Lee S W and Parameswaran M 2007 J. Micromech. Microeng. 17 1093-1098.

[15] Keller S, Haefliger D and Boisen A 2010 J. Micromech. Microeng 20 045024/1-045024/11.

[16] Della Santa A, De Rossi D and Mazzoldi A 1997 Synth. Met. 90 93-100.

[17] Christophersen M, Shapiro B and Smela E 2006 Sens. Actuators, B 115 596-609

[18] Pei Q and Inganäs O 1992 J. Phys. Chem. 96 10507-10514

[19] Alici G, Metz P and Spinks G M 2006 Smart Mater. Struct. 15 243-252 